Bull. Austral. Math. Soc.

47 н 10,54 н $25,54 \mathrm{E} 45$

VOL. 53 (1996) [13-19]

\title{
AN ABSTRACT COMMON FIXED POINT PRINCIPLE AND ITS APPLICATIONS
}

\author{
JACEK R. JACHYMSKI
}

\begin{abstract}
We establish a common fixed point principle for a commutative farnily of self-maps on an abstract set. This principle easily yields the Markoff-Kakutani theorem for affine maps, Kirk's theorem for nonexpansive maps and Cano's theorem for maps on the unit interval. As another application we obtain a new common fixed point theorem for a commutative family of maps on an arbitrary interval, which generalises an earlier result of Mitchell.
\end{abstract}

\section{INTRODUCTION}

There are several common fixed point theorems involving different families of maps, but having similar proofs after all. We mean here the Markoff-Kakutani theorem for affine maps (see for example, [3, p.75]), Kirk's theorem for nonexpansive maps (see for example, $[6$, p.485-486]) and a theorem of Cano [1, Theorem 1] for maps on the unit interval. Our purpose here is to establish a common fixed point principle, which will subsume each of the above mentioned results. This is done in the next section (see Theorem 1). We want to emphasise that our principle involves maps on an abstract set equipped with neither topology nor order structure. Nevertheless, our Theorem 1 combined with a suitable fixed point result for a single map enables us to obtain the above theorems as immediate corollaries. Furthermore, it gives us also the possibility to get some new results in this direction, such as, for example, an extension of Theorem 3 of Mitchell [5] to a family of maps on an arbitrary (not necessarily compact) interval (see Theorem 2). As a by-product of the proof of Theorem 2, we give a characterisation of the set of periodic points of a map, for which the family of its iterates is equicontinuous. This improves upon the recent results of Liu [4].

The letter $\mathbf{N}$ denotes the set of all positive integers. For a map $g$ and $n \in \mathbf{N}$, $g^{n}$ is the $n$th iterate of $g, F(g)$ and $P(g)$ denote the sets of all fixed points and all periodic points of $g$, respectively (a point $x$ is a periodic point of $g$ if $x=g^{n}(x)$ for some $n \in \mathrm{N}$ ). The letters $I$ and $J$ denote the unit interval and an arbitrary interval, respectively. A set $A$ is said to be $g$-invariant if $g(A) \subseteq A$.

Received 8th March, 1995

Copyright Clearance Centre, Inc. Serial-fee code: 0004-9729/96 \$A2.00+0.00. 


\section{A COMMON FIXED POINT PRINCIPLE}

Let $X$ be a nonempty set and $\Sigma$ be a family of its subsets. We begin with the following defiintion.

Definition 1: A family $\Sigma$ has the property (P) if and only if $\emptyset \in \Sigma, X \in \Sigma$ and for any sets $A, B$ in $\Sigma, A \cap B \in \Sigma$.

For a family $\Sigma$ with the property $(\mathrm{P})$, we define the class $\mathcal{F}(\Sigma)$ of self-maps of $X$ by

$$
g \in \mathcal{F}(\Sigma) \quad \text { if and only if } \quad F(g) \neq \emptyset, F(g) \in \Sigma,
$$

and for any nonempty $g$-invariant set $A \in \Sigma, \quad F(g) \cap A \neq \emptyset$.

To illuminate the above notions, we give a few examples of families $\Sigma$ and corresponding classes $\mathcal{F}(\Sigma)$.

EXAmple 1. Let $X:=I$ and $\Sigma:=\{\emptyset\} \cup\{[a, b]: a, b \in I, a \leqslant b\}$. Following Cano [1] define the class $\mathbf{C}_{1}$ by

$$
\mathrm{C}_{1}:=\{g: I \mapsto I \mid g \text { is continuous and } F(g) \text { is a closed interval }\} \text {. }
$$

Let $g: I \mapsto I$ be continuous. It is easy to verify that $g \in \mathcal{F}(\Sigma)$ if and only if $g \in \mathbf{C}_{1}$.

ExAmple 2. Let $X:=I$ and $\Sigma$ be the family of all closed subsets of $I$. Following Cano [1] define the class $\mathbf{C}_{2}$ by

$$
\mathrm{C}_{2}:=\{g: I \mapsto I \mid g \text { is continuous and } F(g)=P(g)\}
$$

Let $g: I \mapsto I$ be continuous. Then $g \in \mathcal{F}(\Sigma)$ if and only if $g \in \mathbf{C}_{2}$. This equivalence follows from Theorem 1 [2].

EXAMPLE 3. Let $X$ be a nonempty closed bounded and convex set in a uniformly convex Banach space. Let $\Sigma$ be the family of all closed and convex subsets of $X$. It follows from the Browder-Göhde-Kirk Theorem (see, for example, [6, p.478]), that $\mathcal{F}(\Sigma)$ contains the family of all nonexpansive self-maps of $X$.

EXAMPLE 4. Let $X$ be a nonempty compact convex set in a locally convex topological space. Let $\Sigma$ be the family of all closed and convex subsets of $X$. It follows from the Schauder-Tychonoff Theorem (see for example, $[3$, p.74]), that $\mathcal{F}(\Sigma)$ contains the family of all continuous affine self-maps of $X$.

The main result is preceded by the following useful lemma. 
LemMa 1. Let $X$ be a nonempty set and $\Sigma$ be a family of its subsets possessing the property $(P)$. If $\mathcal{F} \subseteq \mathcal{F}(\Sigma)$ is a commutative family of self-maps of $X$ then the family $\{F(g): g \in \mathcal{F}\}$ has the finite intersection property.

Proof: Fix a positive integer $n$ and let $g_{i} \in \mathcal{F}$ for $i=1, \ldots, n$. We must prove that $\bigcap_{i=1}^{n} F\left(g_{i}\right) \neq \emptyset$. We shall apply induction with respect to $k$ to show that for all $k \in 1, \ldots, n$,

$$
C_{k}:=\bigcap_{i=1}^{k} F\left(g_{i}\right) \neq \emptyset
$$

in particular, $\bigcap_{i=1}^{n} F\left(g_{i}\right) \neq \emptyset$. By the definition of $\mathcal{F}(\Sigma)$, (1) holds if $k=1$. The case when $n=1$ is trivial. So let $n \geqslant 2$. Assuming (1) to hold for some $k, 1 \leqslant k \leqslant n-1$, we shall prove it for $k+1$. By commutativity, we obtain that $g_{k+1}\left(F\left(g_{i}\right)\right) \subseteq F\left(g_{i}\right)$ for $i=1, \ldots, k$, which immediately yields that $C_{k}$ is $g_{k+1}$-invariant. By the definition of $\Sigma$, we may infer that $C_{k} \in \Sigma$, since $F\left(g_{i}\right) \in \Sigma$ for $i=1, \ldots, k$. Further, $C_{k}$ is nonempty by our induction hypothesis. Therefore, using the definition of $\mathcal{F}(\Sigma)$, we obtain that $F\left(g_{k+1}\right) \cap C_{k} \neq \emptyset$, that is, $C_{k+1} \neq \emptyset$, which completes the induction.

THEOREM 1. Let $X$ be a nonempty set, $\Sigma_{1}$ and $\Sigma_{2}$ be families of subsets of $X$ with the property $(P)$, and $\Sigma_{1} \subseteq \Sigma_{2}$. Further, let $f: X \mapsto X$ satisfy the following condition:

$$
F(f) \neq \emptyset, F(f) \in \Sigma_{2},
$$

and for any nonempty $f$-invariant set $A \in \Sigma_{1}, F(f) \cap A \neq \emptyset$.

If $\mathcal{F} \subseteq \mathcal{F}\left(\Sigma_{1}\right) \cup \mathcal{F}\left(\Sigma_{2}\right) \cup\{f\}$ is a commutative family of self-maps of $X$, then the family $\{F(g): g \in \mathcal{F}\}$ has the finite intersection property.

Proof: Let $n \in \mathbf{N}$ and $g_{1}, \ldots, g_{n} \in \mathcal{F}$. We may assume, without loss of generality, that $n \geqslant 3$ and for some $k, k<n$,

$$
g_{1}, \ldots, g_{k-1} \in \mathcal{F}\left(\Sigma_{1}\right), \quad g_{k}=f, \quad g_{k+1}, \ldots, g_{n} \in \mathcal{F}\left(\Sigma_{2}\right) .
$$

By Lemma $1, A:=\bigcap_{j=1}^{k-1} F\left(g_{j}\right) \neq \emptyset$; moreover, $A \in \Sigma_{1}$. By commutativity, $A$ is $f$-invariant so, by hypothesis, $A \cap F(f) \neq \emptyset$, that is, $B:=\bigcap_{j=1}^{k} F\left(g_{j}\right) \neq \emptyset$. Since $F(f) \in \Sigma_{2}$ and $\Sigma_{1} \subseteq \Sigma_{2}$, we may infer that $B \in \Sigma_{2}$. Define

$$
\Sigma:=\left\{B \cap S: S \in \Sigma_{2}\right\} \quad \text { and } \quad \mathcal{F}^{\prime}:=\left\{\left.g_{j}\right|_{B}: j=k+1, \ldots, n\right\} .
$$


We leave it to the reader to verify that then the assumptions of Lemma 1 are satisfied (with the set $B$ and the family $\mathcal{F}^{\prime}$ substituted for $X$ and $\mathcal{F}$, respectively). In particular, that $F\left(\left.g_{j}\right|_{B}\right)\left(=F\left(g_{j}\right) \cap B\right)$ is nonempty for $j=k+1, \ldots, n$, follows from the definition of $\mathcal{F}\left(\Sigma_{2}\right)$ and the fact that for such $j, B \in \Sigma_{2}$ and $B$ is $g_{j}$-invariant. By Lemma 1, $\bigcap_{j=k+1}^{n} F\left(\left.g_{j}\right|_{B}\right) \neq \emptyset$, that is, $\bigcap_{j=1}^{n} F\left(g_{j}\right) \neq \emptyset$, which completes the proof.

Corollary 1. Let $(X, \tau)$ be a compact topological space and $\Sigma_{1}, \Sigma_{2}$ be families of subsets of $X$ as in Theorem 1. Let $f$ be a continuous self-map satisfying (2). If $\mathcal{F} \subseteq \mathcal{F}\left(\Sigma_{1}\right) \cup \mathcal{F}\left(\Sigma_{2}\right) \cup\{f\}$ is a commutative family of continuous self-maps of $X$, then there is a common fixed point for $\mathcal{F}$.

Proof: Apply Theorem 1 and the compactness argument.

REMARK 1. Corollary 1 immediately yields the Markoff-Kakutani theorem [3, p.75]. To see this, define the family $\Sigma_{1}$ as in Example 4, and put $\Sigma_{2}:=\Sigma_{1}$ and $f:=i d$, the identity map on $X$. In the same way, a common fixed point theorem for nonexpansive maps $[6$, p.485] can be deduced (see Example 3; this time equip the set $X$ with the weak topology). We emphasise that, in both cases, we could apply Corollary 1 thanks to the suitable geometrical and topological properties of a set of fixed points of a single map.

REMARK 2. Theorem 1 of Cano [1] is subsumed by Corollary 1 . To see this, define the families $\Sigma_{1}$ and $\Sigma_{2}$ as in Examples 1 and 2, respectively.

\section{COMMUTING MAPS ON AN ARBITRARY INTERVAL}

In this section we shall apply Theorem 1 to obtain extensions of Cano's Theorem 1 [1] and Mitchell's Theorem 3 [5] by involving self-maps of an arbitrary nonempty interval $J$. Define the following classes of maps on $J$.

$$
\begin{aligned}
& \mathbf{C}_{1}^{\prime}:=\{g: J \mapsto J \mid g \text { is continuous and } F(g) \text { is a nonempty } \\
& \text { compact interval }\}, \\
& \mathbf{C}_{2}^{\prime}:=\{g: J \mapsto J \mid g \text { is continuous and for any nonempty closed } \\
& \text { in } J, g \text { - invariant set } A \subseteq J, F(g) \cap A \neq \emptyset\} .
\end{aligned}
$$

Obviously, if $J=I$ then $\mathbf{C}_{1}^{\prime}=\mathbf{C}_{1}$. Simultaneously, it follows from Theorem 1 [2] that $\mathbf{C}_{2}^{\prime}=\mathbf{C}_{2}$ if $J=I$. However, in case of an arbitrary interval, the condition that $P(g)=F(g)$ need not imply $g \in \mathrm{C}_{2}^{\prime}$. (For example, consider $J:=[0, \infty)$, $g(x):=2 x(x \in J)$, and $C:=\left\{2^{n}: n \in \mathrm{N}\right\}$.)

Now, if we put $X:=J$ and define families $\Sigma_{1}, \Sigma_{2}$ analogously as in Examples 1 and 2 , respectively, then applying Theorem 1 one can easily obtain the following extension of Cano's Theorem 1 [1]. 
Proposition 1. Let $f: J \mapsto J$ be continuous and $\mathcal{F} \subseteq \mathbf{C}_{1}^{\prime} \cup \mathbf{C}_{2}^{\prime} \cup\{f\}$ be a commutative family of continuous maps. If for each $g \in \mathcal{F}, P(g) \neq \emptyset$, then the family $\{F(g): g \in \mathcal{F}\}$ has the finite intersection property.

However, the assumptions of Proposition 1 have the disadvantage that given any particular map $g$, it may be difficult to verify whether $g \in \mathbf{C}_{1}^{\prime}$ or $g \in \mathbf{C}_{2}^{\prime}$. The following theorem has more easily verifiable assumptions; it extends Mitchell's Theorem 3 [5].

THEOREM 2. Let $f: J \mapsto J$ be continuous and $\mathcal{F} \cup\{f\}$ be a commutative family of continuous self-maps of a nonempty interval $J$ and such that for each $g \in \mathcal{F}$, the family $\left\{g^{n}: n \in \mathrm{N}\right\}$ is equicontinuous. Then we have:

(i) the family $\{F(g): g \in \mathcal{F} \cup\{f\}\}$ has the finite intersection property if and only if each map $g \in \mathcal{F} \cup\{f\}$ has a periodic point;

(ii) if there is an $h \in \mathcal{F}$ such that $F(h)$ is nonempty compact then the family $\mathcal{F} \cup\{f\}$ has a common fixed point.

We precede the proof with the following lemma.

LEMma 2. Let $g: J \mapsto J$ be a continuous map such that the family $\left\{g^{n}: n \in \mathrm{N}\right\}$ is equicontinuous. Then $F(g)$ is an interval.

Proof: Suppose, on the contrary, $F(g)$ is not an interval. Then there exist points $a, b \in F(g), a<b$, such that $(a, b) \cap F(g)=\emptyset$. One can use the same argument as in the proof of Theorem $2[1]$ to obtain that $\left\{g^{n}: n \in \mathrm{N}\right\}$ could not be equicontinuous at point $a$ or $b$, a contradiction.

COROLLARY 2. Let $g: J \mapsto J$ be a continuous map such that the family $\left\{g^{n}\right.$ : $n \in \mathrm{N}\}$ is equicontinuous. Then $P(\mathrm{~g})$ is an interval. Hence, $P(\mathrm{~g})$ is either uncountable, or it has at most one element.

ProOF: If $P(g)=F(g)$, the result follows from Lemma 2. So suppose $P(g) \neq$ $F(g)$. This implies that $P(g)$ is nonempty, so $F(g)$ is nonempty; for otherwise, either $g(x)>x$ for $x \in J$, or $g(x)<x$ for $x \in J$, which contradicts that $P(g) \neq \emptyset$. Obviously, for each $k \in \mathrm{N}$, the family $\left\{g^{k n}: n \in \mathrm{N}\right\}$ is equicontinuous so, by Lemma $2, F\left(g^{k}\right)$ is an interval. This easily yields the connectivity of $P(g)$, since $P(g)=\bigcup_{k=1}^{\infty} F\left(g^{k}\right)$, and $\bigcap_{k=1}^{\infty} F\left(g^{k}\right)(=F(g))$ is nonempty.

REMARK 3. Corollary 2 generalises and unifies Theorems 1 and 2 of Liu [4].

Proof of TheOREM 2: We start with part (ii). If such a map exists then, by Lemma 2, $F(h)=[a, b]$ for some $a, b \in J$. By commutativity, $[a, b]$ is $g$-invariant for each $g \in \mathcal{F} \cup\{f\}$. To get the results, it suffices to apply Proposition 1 with $[a, b]$ substituted for $J$ and considering the family $\left\{\left.g\right|_{\{a, b]}: g \in \mathcal{F} \cup\{f\}\right\}$, since by Theorem 2 [1], we have then $\left\{\left.g\right|_{[a, b]}: g \in \mathcal{F}\right\} \subseteq \mathbf{C}_{1}^{\prime}$. 
The necessity part of (i) is trivial. In the proof of the sufficiency part of (i) we may restrict to the case, when $F(g)$ is not compact for each $g \in \mathcal{F}$; for otherwise, we could apply (ii). We shall show that $\mathcal{F} \subseteq \mathbf{C}_{2}^{\prime}$ in this case, so the result will follow from Proposition 1. Note that $F(g)$ is nonempty for each $g \in \mathcal{F} \cup\{f\}$ (see the proof of Corollary 2). Now, fix a map $g \in \mathcal{F}$. In the sequel we consider two cases: the first, for a bounded and half-open interval, and the second, for an unbounded and open interval. The analysis of the remaining cases is similar and we omit it.

$1^{\circ} . J=(a, b)$ where $a<b$. Then $F(g)$ must be of a form $[c, b)$, where $a \leqslant c<b$. The case when $c=a$ is trivial. So assume $a<c$. Then, either $g(x)>x$ for $x \in[a, c)$, or $g(x)<x$ for $x \in[a, c)$; for otherwise, $F(g) \cap[a, c) \neq \emptyset$, a contradiction. Since $g(a)>a$ we get $g(x)>x$ for $x \in[a, c)$. Fix an $x \in[a, c)$. If there exists a $k$ in $\mathrm{N}$ such that $g^{k}(x) \in[c, b)$ then $g^{n}(x)=g^{k}(x)$ for $n \geqslant k$. If not, that is, $g^{n}(x) \in[a, c)$ for $n \in \mathbf{N}$ then $\left\{g^{n}(x)\right\}_{n=1}^{\infty}$ is increasing, hence convergent. Since $\lim _{n \rightarrow \infty} g^{n}(x) \in[a, c] \cap F(g)$, we get $\lim _{n \rightarrow \infty} g^{n}(x)=c$. In both cases $\left\{g^{n}(x)\right\}_{n=1}^{\infty}$ is convergent and $\lim _{n \rightarrow \infty} g^{n}(x) \in J$. This easily implies that $g \in \mathbf{C}_{2}^{\prime}$.

$2^{\circ} . J=(a, \infty)$. Assume $F(g) \neq J ;$ for otherwise that, $g \in \mathbf{C}_{2}^{\prime}$ is trivial. Then the following two cases are possible:

(a) $F(g)=(a, b], a<b$. Then, either $g(x)>x$ for $x>b$, or $g(x)<x$ for $x>b$. Suppose $g(x)>x$ for $x>b$. Then $\left\{g^{n}(x)\right\}_{n=1}^{\infty}$ is increasing, hence either $\lim _{n \rightarrow \infty} g^{n}(x) \in(b, \infty)$, or $\lim _{n \rightarrow \infty} g^{n}(x)=\infty$. But $\lim _{n \rightarrow \infty} g^{n}(x)$ cannot be finite; for otherwise, $\lim _{n \rightarrow \infty} g^{n}(x) \in F(g) \cap(b, \infty)$, a contradiction. Thus we get $\lim _{n \rightarrow \infty} g^{n}(x)=\infty$ for $x>b$. Then, however, $\left\{g^{n}: n \in \mathrm{N}\right\}$ cannot be equicontinuous at point $b$, a contradiction. Therefore, we have $g(x)<x$ for $x>b$. Using a similar argument as in $1^{\circ}$ we infer that $\left\{g^{n}(x)\right\}_{n=1}^{\infty}$ is convergent and $\lim _{n \rightarrow \infty} g^{n}(x) \in J$ for $x \in J$, which implies that $g \in \mathbf{C}_{2}^{\prime}$.

(b) $F(g)=[b, \infty), b>a$. Suppose $g(x)<x$ for $x \in(a, b)$. Then $\left\{g^{n}(x)\right\}_{n=1}^{\infty}$ converges to some $c(x)$ in $[a, b)$. But $c$ cannot be in $(a, b)$, since then it would be a fixed point of $g$. Thus we get $c(x)=a$ for $x \in(a, b)$. Then, however, $\left\{g^{n}: n \in \mathrm{N}\right\}$ would not be equicontinuous at point $b$. Therefore, $g(x)>x$ for $x \in(a, b)$. Hence, for $x \in(a, b)$, either $\left\{g^{n}(x)\right\}_{n=1}^{\infty}$ is constant for sufficiently large $n$, or $\lim _{n \rightarrow \infty} g^{n}(x)=b$. Thus, for all $x \in J,\left\{g^{n}(x)\right\}_{n=1}^{\infty}$ is convergent and $\lim _{n \rightarrow \infty} g^{n}(x) \in J$ so $g \in \mathbf{C}_{2}^{\prime}$.

EXAMPLE 5. Let $J:=(0, \infty), \mathcal{F}:=\left\{g_{k}: k \in \mathbf{N}\right\}$, where $g_{k}(x):=x$ for $0<x<1 / k$, and $g_{k}(x):=1 / k$ for $x \geqslant 1 / k$. It is easy to verify that $\mathcal{F}$ satisfies the assumptions of Theorem 2 and there is no common fixed point for $\mathcal{F}$. On the other hand, according to Theorem 2, the family $\{F(g): g \in \mathcal{F}\}$ has the finite intersection property. 


\section{REFERENCES}

[1] J. Cano, 'Common fixed points for a class of commuting mappings on an interval', Proc. Amer. Math. Soc. 86 (1982), 336-338.

[2] S.C. Chu and R.D. Moyer, 'On continuous functions, commuting functions, and fixed points', Fund. Math. 58 (1966), 90-95.

[3] J. Dugundji and A. Granas, Fixed point theory (Polish Scientific Publishers, Warszawa, 1982).

[4] Z. Liu, 'On continuous maps of the interval', Math. Japon. 38 (1993), 509-511.

[5] T. Mitchell, 'Common fixed points for equicontinuous families of mappings', Proc. Amer. Math. Soc. 33 (1972), 146-150.

[6] E. Zeidler, Nonlinear functional analysis and its applications 1: Fixed-Point Theorems (Springer-Verlag, Berlin, Heidelberg, New York, 1986).

Institute of Mathematics

Technical University of Lódí

Żwirki 36

90-924 Łódź

Poland

e-mail: jachymsk@lodz1.p.lodz.pl 